\title{
Commentary on Hypnosis in the Desensitization of Fears of Dying
}

\section{How Clinicians Learn to Integrate Hypnotic Tactics and Strategies into Psychotherapeutic Interventions}

\author{
JOHN F. CHAVES ${ }^{\text {a,b }}$ \\ ${ }^{a}$ School of Dental Medicine, State University of New York at Stony Brook \\ ${ }^{\mathrm{b}}$ Correspondence concerning this article should be addressed to John F. Chaves, Ph.D., \\ Vice Dean for Dental Education and Professor of Dental Medicine, School of Dental Medicine, \\ State University of New York at Stony Brook, Stony Brook, NY 11794-8704 \\ Email: jfchaves@comcast.net
}

\begin{abstract}
Therapeutic suggestions administered within a hypnotic context can improve the efficacy of psychotherapeutic interventions in dealing with a wide range of clinical problems. Hamburg (2006) illustrates how he overcame both theoretical barriers and tactical issues in treating two clients who presented with fear of dying. His account calls attention to the need to help clinicians make the transition from introductory hypnosis training programs to actually applying hypnotic tactics and strategies in the clinical context. The development of clinical and experimental hypnosis and behavior therapy have influenced each other more profoundly than has generally been acknowledged. Theoretical barriers to the use of hypnosis in behavioral therapies are more apparent than real.
\end{abstract}

Key words: hypnosis; hypnotherapy; cognitive-behavioral therapy; hypnosis training

Hamburg (2006) has presented us with two very interesting cases in which he applied hypnotic strategies in the course of desensitizing clients who presented with complaints of preoccupation and debilitating fear of death and dying. The use of hypnotic interventions to augment desensitization protocols is not new of course. And as Kirsch and his colleagues have noted ( Kirsch, Montgomery \& Sapirstein, 1995), there are reasons to believe that hypnotic interventions augment the efficacy of these interventions. The cases presented by Hamburg should be of particular interest to clinicians who are new to hypnosis because they reveal very clearly how he analyzed these cases and how his use of hypnosis was predicated on a flexible theoretical models that were driven by current thinking about hypnosis and about behavior therapy. In some of my own previous writings I have also discussed the utility of viewing hypnosis as a platform suitable for launching a variety of therapeutic interventions derived from alternative theoretical perspectives (Chaves, 1997a; Brown \& Chaves, 1980). 
One of the most instructive aspects of Hamburg's paper relates to the process by which he was introduced to the topic of hypnosis and eventually found a way to introduce hypnotic techniques into his clinical practice. Like many clinicians, Hamburg learned hypnosis many years after completing his formal training program, by taking one of the standard introductory workshops offered by one of the recognized professional societies. My own experience in teaching similar workshops is that participants commonly develop a high level of interest in the subject during the workshop, but frequently do not make the transition to actually using the techniques in practice unless they pursue intermediate workshops, or have experienced colleagues nearby to offer the needed encouragement and supervision when appropriate.

This apparent reluctance to incorporate hypnotic techniques into clinical practice is not restricted to psychotherapists. Other health processionals, including dentists and physicians who take such introductory workshops are similarly hesitant. Within the last decade I taught at a large Midwestern dental school for eight years and developed a new hypnosis elective for fourth-year dental students and residents. In the course of trying to identify dental clinicians who might be interested in participating in the course, I was surprised to discover that 17 of my colleagues had taken formal introductory workshops in hypnosis, but that none of them had actually used the procedures clinically. Nevertheless, most remained interested in the topic and, collectively, they held more positive attitudes towards hypnosis than faculty, dental students, or their patients, who had not had such exposure (Chaves 2004). Clearly those of us who recognize the advantages conferred by the use of hypnosis and advocate for its wider clinical application must be more concerned about easing the transition from the theoretical knowledge gained in workshops to actual clinical application. The recent appearance of some excellent, evidence-based texts and clinical handbooks on the topic should help (e.g. Lynn, Kirsch, \& Rhue, 1997; Kirsch \& Lynn, 2005; Kirsch, Capafons, Cardena-Buelna, \& Amigo, 1998), but other strategies may be needed.

Hamburg (2006) is undoubtedly correct in identifying some of the barriers that have interfered with the wider integration of hypnosis into clinical practice, such as disagreement about the nature of hypnosis and the widespread use of mentalistic constructs (Chaves, 1997b). Nevertheless, some well-known early behavior therapists, beyond those mentioned by Hamburg, candidly acknowledged the indebtedness of behavioral therapy to hypnosis (e.g. Cautela, 1966, Krasner, 1971). Sometimes research aimed at clarifying the mechanisms by which suggestion (with or without hypnosis) achieved its gains has been adopted by those working in the domain of behavior therapy.

My own early research on cognitive strategies for pain management began as an effort to understand the differential efficacy of certain kinds of hypnotic suggestions in attenuating experimental pain (e.g. Chaves \& Barber, 1975). In turn, that research led to the identification of a subset of individuals who spontaneously engage in what may be viewed as a kind of spontaneous negative self-hypnotic procedure that I labeled "catastrophizing," which appears to exacerbate pain and suffering during acute and chronic pain (Brown \& Chaves, 1980; Chaves \& Brown, 1987; Brown, Chaves, \& Leonoff, 1981). This process is the inverse counterpart of the uncritical acceptance of the positive suggestions and images that are a part of most therapeutic 
and Strategies into Psychotherapeutic Interventions

J.F. Chaves

Pragmatic Case Studies in Psychotherapy, http://pcsp.libraries.rutgers.edu

Volume 2, Module 2, Article 3, pp. 1-5, 05-11-06 [copyright by author]

protocols used in the hypnotic context. Hamburg (2006) nicely illustrates this with his emphasis on the routine inclusion of generalized suggestions emphasizing the possibility of rapid change along with client-specific suggestions. In recent years, management of the process of catastrophizing has been seen as fundamental in cognitive-behavioral approaches to pain management and a pivotal construct in understanding and managing chronic pain (e.g., Sullivan, Thorn, Haythornthwaite, Keefe, Martin, Bradley, Lefebvre, 2001.) Hypnotic interventions can play a key role in providing a kind of cognitive prosthesis for catastrophizing patients who seem to lack the ability to engage in such spontaneous coping strategies.

Hamburg (2006) is probably correct that mentalistic constructs, which were employed by some hypnosis researchers and clinicians and which provided the central themes for many hypnosis workshops during the early behavior therapy period, made it easier for many of the early behavior therapists to ignore this literature, especially in the days before cognitive psychology was emerging as a significant new paradigm for psychology. Nevertheless, T. X. Barber, and T. R. Sarbin and their colleagues were writing about hypnosis in a social-behavioralcognitive context prior to the emergence of behavior therapy. Moreover, it was evident that their analyses went far beyond viewing the impact of psychosocial variables as merely an "artifact" as Hamburg suggests.

Traditional hypnotic state theorists like Orne (1959) attempted to distinguish what he saw as the dynamic "essence" of hypnosis from "artifacts" created by the operation of social psychological variables that were not the focus of his interest. Later research clearly showed that Orne's distinction obscured much more than it revealed and was itself fundamentally flawed (Johnson, Maher, \& Barber, 1972). The problem is reminiscent of the pharmacologist, for whom the placebo effect may well be described as an artifact. But for the psychologist, the complex interaction of attitudes, expectations, belief, subtle communications, and so forth that underlies this powerful phenomenon can not be dismissed as mere artifact. Rather they suggest that we routinely fail to appreciate how powerful these psychosocial variables can contribute to adaptive change and human potentialities (Barber, Spanos \& Chaves, 1974; Spanos \& Chaves, 1989).

Turning briefly to strategic considerations, Hamburg is quite correct to point out that ambivalence about a positive clinical outcome can seriously undermine achieving success when applying hypnosis in the clinical setting. However, limiting one's application to the treatment of anxiety disorders and chronic pain does not provide meaningful protection from the pervasive effects of secondary gain. Nowhere is this more obvious than in those cases of chronic pain resulting from accidents that are still under litigation. Creative strategies are often required to deal with these issues, sometimes including the paradoxical withholding of hypnosis for pain pending demonstrable gains in other areas (e.g. Chaves, 1993; 1994 ).

There is much to be learned from Hamburg's candid account of the struggle he went through in formulating therapeutically relevant suggestions for the cases he present. His emphasis on using the clients' own phenomenology of their distress as a foundation for establishing appropriate therapeutic suggestions is entirely appropriate. The approach can be 
thought of as an extension of Spanos' concept of goal-directed fantasy as a critical element in the formulation of hypnotic suggestion (Spanos, 1971). The essence of the concept is encouraging clients to imagine a situation, however counterfactual, that if real, would be consistent with the desired clinical outcome. The essence of the hypnotic context is that it helps to establish positive expectations in a situation in which the acceptance of counterfactual suggestions is understood to be normative. This is the expectation that many clients already bring to the hypnotic context, and is strongly reinforced during the preparation for the hypnotic intervention.

Most behaviorally-oriented clinicians will not be theoretically constrained from adopting hypnotic techniques as evidence continues to mount of their efficacy in dealing with a wide range of significant clinical problems. Increasingly, the evidence supporting its inclusion has been growing and becoming more robust (e.g. Chaves, 2000; in press). Contributions like Hamburg's (2006) make an important contribution by modeling the kind of process clinicians new to hypnosis can adopt as they make the transition from novice to skilled hypnotherapists.

\section{REFERENCES}

Barber, T. X., Spanos, N. P. \& Chaves, J. F. (1974) Hypnotism, imagination \& human potentialities. New York: Pergamon Press.

Brown, J. \& Chaves, J. F. (1980). Hypnosis in the treatment of sexual dysfunction. Journal of Sex and Marital Therapy, 6, 63-74.

Brown, J., Chaves, J. F. \& Leonoff, A. (1981, August) Spontaneous cognitive strategies in two groups of chronic pain patients. Presented to the American Psychological Association, Los Angeles.

Cautela, J. R. (1966) Hypnosis and behavior therapy. Behavior Research and Therapy, 4, 219224.

Chaves, J. \& Brown, J. (1987). Spontaneous cognitive strategies for the control of clinical pain and stress. Journal of Behavioral Medicine, 10, 263-275.

Chaves, J. F. (1993). Hypnosis in pain management (pp. 511-532). In Rhue, J., Lynn, S. J. \& Kirsch, I. (Eds.) Handbook of clinical hypnosis. Washington, D.C.: American Psychological Association.

Chaves, J. F. (1994). Recent advances in the application of hypnosis to pain management. American Journal of Clinical Hypnosis., 37, 117-129.

Chaves, J. F. (1997a). Hypnosis as a "platform" for psychological interventions in pain management. Society for Clinical \& Experimental Hypnosis, $48^{\text {th }}$ Annual Meeting, Washington, DC.

Chaves, J. F. (1997b). The state of the "State" debate in hypnosis: A view from the cognitivebehavioral perspective. International Journal of Clinical and Experimental Hypnosis, 45, 251-265.

Chaves, J. F. (2000). Hypnosis in the management of anxiety associated with medical conditions and their treatment. In D. Mostofsky and D. Barlow (Eds.) Managing the anxiety 
associated with medical conditions and their treatment (pp. 119-147). New York: Allyn \& Bacon.

Chaves, J. F. (2004, August). Hypnosis in the health care setting: Hopes, beliefs, and experience. Invited Address. Annual meeting of the American Psychological Association, Honolulu, HI.

Chaves, J. F. (in press). Hypnosis in the management of chronic pain conditions and the acute pain accompanying their treatment. In D. Beltrutti, A. Lamberto, D. Niv, \& S. Kreitler (Eds.), Chronic pain: The physiological and psychosocial dimensions of diagnosis, assessment, and therapy. Hauppauge, New York: Nova Science.

Hamburg, S.R. (2006). Hypnosis in the desensitization of fears of dying. Pragmatic Case Studies in Psychotherapy [Online], Vol. 2(2), Article 1. Available: http://hdl.rutgers.edu/1782.1/pcsp_journal.

Johnson, R. F. Q., Maher, B., \& Barber, T. X. (1972). Artifact in the "essence of hypnosis": An evaluation of trance logic. Journal of Abnormal Psychology, 79, 212-20.

Kirsch, I., Montgomery, G., Sapirstein, G. (1995). Hypnosis as an adjunct to cognitivebehavioral psychotherapy: A meta-analysis. Journal of Consulting and Clinical Psychology, 63(2): 214-20.

Kirsch, I., Capafons, A., Cardeña-Buono, E., \& Amiga, S. (Eds.). (1998). Clinical hypnosis and self-regulation. Washington, DC: American Psychological Association

Lynn, S., Kirsch, I. \& Rhue, J. W. (Eds.). (1996). Casebook of clinical hypnosis. Washington, DC: American Psychological Association.

Orne, M. T. (1959). The nature of hypnosis: Artifact and essence. Journal of Abnormal \& Social Psychology, 58, 277-299.

Rhue, J., Lynn, S. J. \& Kirsch, I. (1993) (Eds.). Handbook of clinical hypnosis. Washington, D.C.: American Psychological Association

Spanos, N. P. (1971). Goal-directed fantasy and the performance of hypnotic test suggestions. Psychiatry, 34, 86-96.

Spanos, N. P. (1983) The hidden observer as an experimental creation. Journal of Personality and Social Psychology, 44, 170-176.

Spanos, N. P. \& Chaves, J. F. (Eds.). (1989). Hypnosis: The cognitive-behavioral perspective. Buffalo, NY: Prometheus Press.

Sullivan, M.J.L., Thorn, B., Haythornthwaite, J.A., Keefe, F., Martin, M., Bradley, L.A., \& Lefebvre, J.C. (2001). Theoretical perspectives on the relation between catastrophizing and pain. Clinical Journal of Pain, 17, 52-64. 